\title{
Brain Activity and Upper Limb Movement Analysis in Individuals With Down Syndrome Undergoing Transcranial Direct Current Stimulation Combined With Virtual Reality Training: Study Protocol for a Longitudinal Randomized Controlled Trial.
}

JAMILE LOPES ( $\nabla$ jamilepalma@yahoo.com.br)

Faculdade de Ciências Médicas da Santa Casa de São Paulo https://orcid.org/0000-0003-1861-0790 Isabela Marques Miziara

Universidade Federal de Uberlandia

Danial Kahani

University of Strathclyde

Rodolfo Borges Parreira

Faculdade de Ciências Médicas da Santa Casa de São Paulo: Faculdade de Ciencias Medicas da Santa Casa de Sao Paulo

Roberta Delasta Lazzari

Faculdade de Ciências Médicas da Santa Casa de São Paulo: Faculdade de Ciencias Medicas da Santa Casa de Sao Paulo

\section{Lucas Villalta Santos}

Faculdade de Ciências Médicas da Santa Casa de São Paulo: Faculdade de Ciencias Medicas da Santa Casa de Sao Paulo

\section{Carlos Bandeira de Mello Monteiro}

USP: Universidade de Sao Paulo

\section{Deborah Carvalho da Silva Cardoso}

UniEVANGÉLICA Centro Universitário de Anápolis: UniEVANGELICA Centro Universitario de Anapolis Juliana de Oliveira Hassel Mendes

UniEVANGÉLICA Centro Universitário de Anápolis: UniEVANGELICA Centro Universitario de Anapolis Vera Lucia dos Santos Alves

Faculdade de Ciências Médicas da Santa Casa de São Paulo: Faculdade de Ciencias Medicas da Santa Casa de Sao Paulo Iransé Oliveira Silva UniEVANGÉLICA Centro Universitário de Anápolis: UniEVANGELICA Centro Universitario de Anapolis Luis Vicente Oliveira 
Faculdade de Ciências Médicas da Santa Casa de São Paulo: Faculdade de Ciencias Medicas da Santa Casa de Sao Paulo

\section{Bernard Arthur Conway}

University of Strathclyde

\section{Manuela Galli}

POLIMI: Politecnico di Milano

\section{Veronica Cimolin}

POLIMI: Politecnico di Milano

\section{Claudia Santos Oliveira}

Faculdade de Ciências Médicas da Santa Casa de São Paulo: Faculdade de Ciencias Medicas da Santa Casa de Sao Paulo

\section{Study protocol}

Keywords: Down Syndrome, Brain Activity, Virtual Reality, Transcranial Direct Current Stimulation, upper limb movement

Posted Date: April 28th, 2021

DOI: https://doi.org/10.21203/rs.3.rs-462899/v1

License: (c) (i) This work is licensed under a Creative Commons Attribution 4.0 International License. Read Full License 


\section{Abstract}

Background: Children with Down Syndrome have poorer functional and sensory skills compared to children with typical development. Virtual reality training could help improve these skills. Moreover, transcranial direct current stimulation has achieved promising results in terms of enhancing the effects of physical and sensory therapy by modulating cortical excitability.

Methods/design The sample will be defined after conducting a pilot study with the same methodology as that to be used in the main study. The study will involve individuals with Down Syndrome with a cognitive age of six to 12 years according to the Wechsler Abbreviated Intelligence Scale. Will be randomly allocated into two groups: Group 1 AtDCS combined with a virtual reality game and a manual motor task. Group 2 sham AtDCS with a virtual reality game and a manual motor task. The training protocol will involve 10 sessions of AtDCS during memory and motor task games. Three 20-minute sessions will be held per week for a total of 10 sessions. Evaluations will be performed on three different occasions: preintervention, post-intervention (after session 10) and follow-up (one month after the proposed training). The evaluations will consist of an analysis of electroencephalographic signals, electromyographic signals of the biceps and triceps brachii and the three-dimensional reconstruction of the reaching movement. The results will be analyzed statistically, with the significance level set at $5 \%(p \leq 0.05)$.

Discussion: The optimization of the results obtained is believed to be related to interactive training with a wide range of activities and scenarios involving multiple sensory channels and the creation of exercises, the intensity of which can be adjusted to the needs of individuals. Therefore, the proposed study aims to complement the literature with further information and different variables to provide the scientific community with clinical data on this combination of interventions.

Trial Registration: REBEC protocol number RBR-43pk59 registered on 2019/03/27 and Human Ethics Committee number 3.608.521 approved on 2019/09/30.

\section{Background}

Down syndrome (SD) that according to the International Classification of Diseases (ICD-10) receives the code Q - 90 is a genetic condition considered one of the most frequent numerical autosomal chromosome anomalies and is recognized as a major cause of mental disability. ${ }^{[1,2]}$ This population exhibits a variety of learning and developmental problems, which exert a direct impact on selective motor control, compromising the acquisition of motor skills and functional independence. ${ }^{[3-5]}$

The literature describes several morphofunctional characteristics that affect the motor system in individuals with DS, such as muscle hypotonia (99\%), broad hands and short fingers (70\%), joint hyperextension $(80 \%)$ and ligament laxity, which is correlated with joint instability. These problems are related to delayed motor development and muscle diastasis, which hinder the performance of precise movements. ${ }^{[5]}$ Although these characteristics are not fully understood in the literature, children with DS are known to have structural and functional impairments of the brain in the early stages of development, 
with deficiencies in both gray and white matter, reduced myelinization as well as presynaptic and synaptic disorders. ${ }^{[5]}$ These problems may influence the initial development of brain circuits, affecting the installation and consolidation of the neural network connections necessary to establish the mechanisms of attention, memory, correlation and abstract thinking. ${ }^{[11,12]}$

The magnitude of motor and cognitive deficits in this population varies throughout development ${ }^{[6]}$ and can interfere with the ability to perform routine functions and tasks in an independent manner. ${ }^{[7-10]}$ Thus, novel rehabilitation techniques have been studied with the aim of minimizing the dysfunctions that affect this population. The results of clinical studies involving transcranial direct current stimulation have shown considerable potential in the treatment of neurological disorders ${ }^{[1-16]}$ by modulating the excitability of the central nervous system. ${ }^{[17-18]}$

tDCS is a relatively low-cost technique that is easy to apply, has minimal adverse effects and is capable of inducing lasting changes in the excitability of the motor cortex. ${ }^{[17]}$ Greater benefits are seen when this technique is combined with a motor task. According to the literature, tDCS promotes changes in the dysfunctional excitability pattern, enabling motor training to mold the functional pattern of cortical activity ${ }^{18}$ by activating task-specific neural networks, thereby optimizing the functional outcome due to the enhancement of neuroplastic changes. ${ }^{[18-21]}$

Virtual reality (VR) stands out among the most widely used motor training methods in the literature. ${ }^{[22]}$ Rehabilitation-oriented VR games have gained prominence by enabling greater interactivity and immersion into the rehabilitation process compared to conventional treatments. Games create alternative scenarios, encouraging the practice of physical activity that might otherwise be considered tedious and tiresome. ${ }^{[22-26]}$

Although the use of VR therapies for the treatment of movement dysfunctions in individuals with DS has been studied in the academic community, there remains a lack of scientific material on the subject. ${ }^{[27-28]}$ However, studies involving the use of VR for upper limb training in children with cerebral palsy have shown promising results, such as improved sensorimotor and adaptive information. ${ }^{[29-30]}$ VR as an auxiliary instrument in physical therapy provides a motivational, playful manner to facilitate the development of sensory and motor skills, favoring the active participation of the individual, the training of planning skills, motor control and the development of strategies for overcoming motor challenges.

This will be the first study to correlate variables and evaluate the effects of anodal tDCS (AtDCS) combined with physical therapy involving a VR game in children with DS. For such, it is necessary to employ evaluative methods that can determine the effects of this combined therapy on the population with DS. For a complete evaluation of motor adaptation and the effects of the proposed intervention, electromyographic signals, electroencephalographic signals and movement kinematics will be obtained during a manual reaching task motivated by a VR game. 


\section{Methods / Design}

The objective of the study is to perform a comparative analysis of brain activity, muscle activity and upper limb kinematics during the execution of a manual motor task motivated by a VR game during AtDCS in individuals with DS. Thus, psychomotor, muscular and cognitive skills will be assessed pre, post, and follow-up intervention. The objectives of the study will be to (i) correlate variables related to movement kinematics, motor activity and brain activity, (ii) compare the effects of transcranial stimulation in individuals with DS and those with typical development (TD), (iii) determine the effects and possible adverse effects of AtDCS at an intensity of $1 \mathrm{~mA}$ administered during 20 minutes of training in individuals with DS and (iv) determine the effects of different AtDCS montages in individuals with DS.

The null hypothesis of the study is that the results of ten sessions of AtDCS over the F3 region of the cortex during a motor task motivated by a VR game will not differ significantly from the results obtained from ten sessions of sham tDCS with the same training in individuals with DS. The alternative hypothesis is that the results of ten sessions of AtDCS over the F3 region of the cortex during a motor task motivated by a VR game will differ significantly from the results obtained from ten sessions of sham tDCS with the same training in individuals with DS.

\section{Ethical aspects}

The present study complies with the norms governing research involving human subjects stipulated by the Brazilian National Board of Health in October 1996 and updated in Resolution 466 in 2012 as well as the precepts delineated in the Declaration of Helsinki. The study received approval from the Research Ethics Committee of the School of Medical Sciences of the Santa Casa de São Paulo (certificate number: 3.608.521) and REBEC protocol number RBR-43pk59 registered on 2019/03/27, the authors confirm that all ongoing and related trials for this drug/intervention are registered. All volunteers and their legal guardians will agree to participate in the study by signing a statement of informed consent. The participants will be allowed to abandon the study at any time with no negative consequences. Absolute confidentiality regarding the identification of individuals will be assured. The participants will be informed of the existence of a sham group prior to the start of the survey. The sham intervention procedures will always be performed in combination with active (VR training), reducing impact on the patient.

\section{Study design}

This is a protocol study for a longitudinal randomized controlled clinical trial consisting of three studies (Fig 1) and written based on the SPIRIT. The time schedule of the trial enrollment, interventions, assessments and visits of participants is displayed in Table 1.

\section{Sample selection and characterization of healthy individuals}


Individuals with typical development will be recruited from a database at the university. The inclusion criteria are no diagnosis of cognitive and/or neuromotor disorders, cooperation during the procedures and a signed statement of informed consent.

\section{Sample selection and characterization individuals with DS}

The population will be composed of individuals diagnosed with DS who will be recruited from the outpatient clinic of the University Center of Anápolis and the Association of Parents and Friends of Exceptional Children of Anápolis, which is a partner institution of the university. The inclusion criteria are a diagnosis of DS, the ability to understand procedures involved in the study, adequate cooperation, cognitive age between six and 12 years defined by the Wechsler Abbreviated Intelligence Scale (WISC) and a signed statement of informed consent. The exclusion criteria are having undergone surgical procedures in the 12 months prior to the onset of the training sessions, orthopedic deformities of the upper limbs or spine with an indication for surgery, a diagnosis of uncontrolled epilepsy, metal implants in the brain or hearing aids, other neurological disorder besides DS and the use of a pacemaker.

\section{Evaluation of cognitive age}

All individuals with DS will be assessed with regards to cognitive age using the Wechsler Abbreviated Scale of Intelligence (WASI), which is a tool designed to assess intellectual performance. This scale was developed from the Wechsler Intelligence Scale and the revised WISC-R scale for children to meet the demand for a short, reliable intelligence measure that could be used in clinical, psychoeducational and research settings while maintaining the possibility of interpreting a unified instrument. The evaluation using this scale will be performed by the psychologist in charge of the cognitive analysis.

\section{Randomization}

In Study 1, we will perform a stratified permuted block randomization to balance gender and based on individual's conditions, i.e., children with DS and children with typical development using a computergenerated random number at www.randomizacion.com. For Study 2, ten participants will be randomly divided into two groups (experimental and control); each group will consist of five volunteer The randomization method will be performed by a person who will not otherwise participate in the study.

\section{Group allocation}

The allocation to the groups will be performed with opaque and sealed envelopes. After signing the statement of informed consent, the individuals will choose an envelope with the name of the group to which they will be assigned in Studies 2 and 3 . This procedure will be managed by a person who will not otherwise participate in the study.

\section{Blinding}


The researcher who will conduct the evaluations will be blinded to the group assignment and the objectives of the study and will not participate in the intervention protocols.

All electrode placement procedures will be performed in the sham group as well, but the stimulator will be switched on for only 30 seconds and will then be switched off. Thus, the subjects will have the initial sensation of electrostimulation, but will not receive any stimulation throughout the remaining time.

\section{Data management and monitoring}

Data on the participants will be collected during the eligibility assessment. Signed consent forms will be safeguarded. This study will be performed in accordance with the approved protocol. If a participant is excluded or withdraws from the study, the reason for the exclusion will be recorded.

If the participant presents any adverse effect related to the intervention process for example itching, burning sensation, headache, and pain, it will be reported to the ethics committee and procedures will be performed to ensure the participant's safety. However, due to the simplicity and safety of the protocol, we do not expect the participants to experience any adverse events.

\section{Recruitment}

Strategies will be employed to encourage participation in the study and reduce the dropout rate. The individuals with DS will be recruited from the outpatient clinic of the University Center of Anápolis and the Association of Parents and Friends of Exceptional Children of Anápolis. Parents/guardians will be contacted by telephone (or other means of communication). Pamphlets will be created with a brief description of the objectives, type of therapeutic intervention, expected results and eligible population. The researchers of the project will be responsible for this disclosure as well as leaving contact information for the parents of eligible children. The individuals selected for this study will be recruited according to the schedule registered with the ethics committee and REBEC in Dezember 2020.

\section{Study 1}

A cross-sectional study will be performed to determine the pattern of normality between individuals with DS and those with typical development. The sample selected for this study will be in accordance with the eligibility and quantity criteria for a convenience sample. The participants will be divided into two groups and each group will consist of 12 children.

\section{Study 2}

A pilot study will be conducted to determine the sample size for the longitudinal randomized controlled clinical trial. For the proper sample size calculation, the pilot study will be carried out using the same methods as those to be employed in the main study. The pilot study will involve ten children selected based on the eligibility criteria for the individuals with DS. The sample size for the clinical trial will be calculated based on the means obtained in the experimental and control groups of this pilot study, 
considering brain activity as the primary outcome, a unidirectional alpha of 0.05 and a power of $80 \%$. The sample size will be then increased by $20 \%$ to compensate for possible dropouts.

\section{Study 3}

A longitudinal randomized controlled trial will be conducted. The sample will be selected based on the results of the pilot study and the individuals will be randomly distributed into two groups using a randomization website (randomization.com).

Experimental Group: AtDCS (anode placed over F3 of the International 10-20 Electroencephalogram System and the cathode placed over the right deltoid muscle) combined with VR training;

Control Group: Sham tDCS (the same electrode montages as in the experimental group) combined with VR training. At the end of the study, individuals in the sham group will receive the same stimulation protocol as the experimental group as a form of treatment to enable the same gain in functioning that we hope to find in the experimental group.

\section{Evaluation process}

The evaluation process 1 (cross-sectional study), 2 (pilot study) and 3 (clinical trial [pre-intervention, postintervention and one-month follow-up]) will be performed according to Resolution No. 466/12 of the Brazilian National Board of Health. Each evaluation session will last a maximum of one hour and 30 minutes.

The evaluations will be performed at the Motion Analysis Laboratory of the University Center of Anapolis. Brain activity will be measured through the acquisition of electroencephalographic (EEG) signals. Muscle activity will be determined through the acquisition of electromyographic (EMG) signals. Threedimensional motion analysis will be used for the acquisition of the kinematic data.

Initially, the identification form will be completed and the anthropometric data will be collected. The volunteers will be assessed using a non-immersive virtual reality motor task on a Dell S2240T LED 21.5" touch screen monitor. The game will consist of figures displayed in random order. The child will be instructed to touch only the figures indicated in the corner of the screen, performing a reaching motor task combined with a cognitive task of memorization and attention. After each tap (trial), the individual will return the hand to the initial support position and wait for a correct new figure to be displayed.

During the task, the EEG signals, EMG signals and upper limb kinematic data will be collected for the determination of neural signals associated with movement.

Electroencephalographic analysis: Brain activity will be investigated by electroencephalography using the eego $^{\text {TM }}$ sports equipment concomitantly to electromyography and the kinematic analysis. The EEG equipment is a battery-powered system with 64-channel EEG recordings and sampling up to $16 \mathrm{kHz}$. 
EEG is a method for assessing the relationship between the brain and behavior and provides a direct realtime measure of neural activity, identifying critical neural mechanisms for motor performance and facilitating the recognition and modification of mental states associated with particular cortical arousal patterns and concomitant behavioral outcomes. ${ }^{[32-35]}$

The task will be performed in a small room without the possibility of external noise. Only the evaluator and child will be present. The child will be placed in the upright position on a chair adjusted so that the feet rest comfortably on the floor. The child will be seated at a square table on which the hands will rest in the starting position. A memory game will be projected on a touch monitor with possibilities of advancement in difficulty level and the subjective measurement of hits and misses. The volunteer will be oriented to perform a manual motor task, touching the screen to match figures during the memory game. After each touch (trial), the hand will return to the initial position. The task will be performed concomitantly with the EEG reading to identify neural signals associated with the movement.

The data will be preprocessed using the $a s a^{T M}$ software, which is a highly flexible EEG/ERP and magnetoencephalography (MEG) analysis package with a variety of source reconstruction and signal analysis features. ${ }^{[36]}$

EMG Analysis: Activity of the brachial biceps and triceps muscles during the manual motor task will be determined using EMG. Electrical activity resulting from activation of elbow flexors and extensors will be collected using an eight-channel electromyograph (FREEEMG, BTS Engineering) with a bioelectric signal amplifier, wireless data transmission and bipolar electrodes with a 2000-fold total gain and frequency ranging from 20 to $450 \mathrm{~Hz}$. The impedance and common rejection mode ratio of the equipment is $>1015$ $\Omega / 0.2 \mathrm{pF}$ and $60 / 10 \mathrm{~Hz} 92 \mathrm{~dB}$, respectively. The motor point of the muscles will be identified for electrode placement and the skin will be cleaned with $70 \%$ alcohol to reduce bioimpedance, following the guidelines of the Surface Electromyography for Noninvasive Muscle Assessment. ${ }^{37}$ All EMG data will be scanned at 1000 frames per second using the BTS MYOLAB software program.

Threedimensional motion analysis: Upper limb motion kinematics will be evaluated using the SMART-D 140 system (BTS, Milan, Italy) (Fig 4), with eight infrared-sensitive cameras, a sampling frequency of 100 $\mathrm{Hz}$ and video system synchronized with the SMART-D system. Passive markers will be positioned at anatomical landmarks directly on the skin with specific tape following the SMARTup protocol: the experimental setup (Fig 2). ${ }^{[37-40]} A$ total of 18 markers measuring $15 \mathrm{~mm}$ in diameter will be used to identify the position of the head, trunk and upper limbs (arm, forearm and hand).

The data from all equipment (SMART-D 140, BTS, Milan, Italy; ${ }^{[37-41]}$ FREEEMG, BTS Engineering; ${ }^{[36]}$ and eego $^{\text {TM }}$ mylab) will be collected simultaneously during the reaching task with a cognitive component. The participant will be positioned on a chair with his/her arms on the evaluation table in front of a touch monitor, which will display figures in random order. The child will be instructed to touch only the figures 
indicated in the corner of the screen, performing a reaching task combined with a cognitive task of memorization and attention. After each tap (trial), the hand should return to the initial support position and wait for a new correct figure to be displayed (Fig 3).

\section{Intervention protocol}

The therapeutic intervention will only be performed in Studies 2 and 3 of this protocol and will consist of a combination of tDCS and VR. The protocol will follow the safety procedures described in the literature for the use of tDCS in the population with neurological disorders. ${ }^{[42-50]}$ Three 20-min sessions of tDCS concomitant with VR will be held for a total of ten sessions. ${ }^{[44-50]}$. Training is preceded by fitting and adapting the equipment to the participant. During the clinical trial, participants are prohibited initiate therapeutic activities that may be similar in the biomechanics used in the tests and in the intervention. However, it is allowed to start any other activity as long as you inform the researchers responsible for the study previously for biomechanical investigation of the new activity.

Transcranial direct current stimulation (tDCS): Stimulation will be administered using a tDCS device (DCStimulator NeuroConn, Germany) and two sponge (non-metallic) surface electrodes measuring $25 \mathrm{~cm}^{2}$ (5 $\times 5 \mathrm{~cm}$ ) soaked in saline. The participants will be randomly allocated to two types of treatment: (1) active AtDCS; and (2) sham tDCS. The anode will be positioned over F3 of the international 10-20 electroencephalogram system and the cathode will be positioned over the right deltoid muscle. This montage (Fig 4) will enable the administration of AtDCS over the dorsolateral prefrontal cortex (DLPFC) while minimizing the effect of cathodal stimulation of the brain. The $1 \mathrm{~mA}$ current (current density: 0.029 $\mathrm{mA} / \mathrm{cm}^{2}$ ) will be administered over the DLPFC for 20 minutes during upper limb training. The stimulator has a button that allows the operator to control the intensity of the current. Stimulation will be gradually increased to $1 \mathrm{~mA}$ at the beginning of the session and gradually decreased during the final $10 \mathrm{~s}$ of the session. Sham stimulation will consist of the same electrode montage and the stimulator will be turned on for $30 \mathrm{~s}$, giving the child the initial sense of stimulation, but no current will be delivered for the remainder of the session. This is considered a valid control procedure in studies involving tDCS. ${ }^{[42-50]}$ The participants will undergo a rehabilitation program while receiving tDCS. The rehabilitation program is based on the serial reaction-time task, which has been used extensively in upper limb studies. During the experiment, the participants will be seated on a chair in front of a touch screen monitor, head and arms supported (ankle angle: $\sim 110^{\circ}$; knee angle: $\sim 150^{\circ}$; hip angle: $\sim 120^{\circ}$ ). The participants will be instructed to avoid drinking coffee or consuming other stimulants on the day of the trial.

Adverse effects: Potential adverse effects of tDCS will be assessed at the end of each session using a questionnaire administered to the child. The questionnaire will address the perception of symptoms that occurred during the session, such as tingling, itching, burning sensation, headache, pain at the electrode site, drowsiness and altered mood. The participants will be instructed to respond using a three-point scale. Caregivers and children will also receive open-ended questions at the beginning of each session addressing the occurrence of headache, scalp pain, itching, burning sensation, redness of the skin, drowsiness, difficulty concentrating and mood swings between sessions. 


\section{Virtual reality}

Training sessions combined with tDCS will be held three times a week on non-consecutive days. Each session will last 20 minutes. Two training sessions will be held before the start of the intervention protocol to familiarize the participants with the procedures. Records will be made of the number of sessions attended, the duration of each session and the final score of the game based on the number of correct and incorrect taps on the screen. The entire training protocol will be carried out by a project employee with interests independent of the objective of the study.

The game will consist of five balls displayed on a touch screen. When the game starts, one of the balls will change color. At that moment, the child must touch the respective ball. After the child touches the screen, the ball returns to its original color and another ball will change color after a three-second interval. The game will consist of seven blocks: R1, R2, S1, S2, S3, R3 and S4. R blocks are those with random sequences and $S$ blocks are those with repetitive sequences, with each block consisting of 20 sequences. A sequence is the order in which the balls change color. The sequences are either randomized or repetitive, depending on the block. Each sequence will be composed of five touches, such that 100 touches are performed by the end of each block.

Among the parameters obtained from the game for the analysis of the learning process in children with DS, the error will be calculated. The error is obtained by calculating the distance between the center of the ball and the region touched as well as the time interval between the moment that the ball changes color and the child's touch. The total error is given by the sum of the time interval and the distance between the points (touch and center of the ball) for each event.

To ensure the synchronization of the game, the acquisition of the EEG signals, the motion reconstruction and the EMG system, it will be necessary for a pulse to be sent with each child's touch. This pulse will be interpreted by an Arduino platform, which will subsequently send the information to both systems. ${ }^{\text {[51-52] }}$

\section{FREQUENCY OF ANALYSES}

Result data will be analyzed only once, at the end analysis of each study.

\section{Statistical Analysis Of Results}

Statistical analysis will involve intention-to-treat analysis. If data are lost during the course of the study, the analysis will be performed by carrying the results of the last observation forward to adjust for missing data in post-intervention assessments. The Shapiro-Wilk normality test will be used to evaluate the distribution of data regarding adherence to the Gaussian curve. Parametric variables will be expressed as mean and standard deviation values. Nonparametric variables will be expressed as median and interquartile range. The two intervention groups will be analyzed for differences regarding anthropometric, clinical and functional variables using the chi-square test for categorical variables and the t-test for continuous variables. Mean, median, standard deviation, minimum and maximum values 
will be used for the descriptive analysis of the effects of tDCS and VR on the brain, muscle and movement variables (quantitative variables). Absolute (n) and relative (\%) frequencies will be used for qualitative variables. To evaluate the quantitative variables measured over time (pre-intervention, postintervention and one-month follow-up) comparing the treatment and control group, we will perform repeated-measures ANOVA. On all tests, a significance level of $5 \%$ will be used. The effect size (Cohen's d) will be calculated from the difference of the values obtained in the pre-intervention, post-intervention and one-month follow-up evaluations, with both intragroup and intergroup comparisons. Predictive response factors will be identified through logistic regression analysis. A p-value $<0.05$ will be considered indicative of statistical significance. The data will be organized and tabulated using the Statistical Package for Social Sciences (v.22.0).

\section{Discussion}

Neurological disorders in children with DS can lead to deficits in perceptual interactions as well as a poorer functional performance ${ }^{8}$ than children without this diagnosis. ${ }^{21,22-25}$ Delays in the acquisition of basic motor skills are common in this population, indicating that such skills emerge at a different time compared to children with typical development. ${ }^{[10,21-22]}$

VR activities constitute a promising resource in the rehabilitation process by promoting the repetition of movements during functional and motor training, the improvement of sensorimotor and adaptive information and the overcoming of difficulties with regards to activities of daily living. ${ }^{[2-53]}$ Moreover, tDCS provides the possibility of optimizing the effects of motor training through a VR game by modulating cortical excitability. According to the literature, the combination of these two techniques is appropriate for this population.

The optimization of the results obtained through VR games is believed to be related to interactive training with a wide range of activities and scenarios involving multiple sensory channels and the creation of exercises, the intensity of which can be adjusted to the needs of individuals with DS. ${ }^{[53-55]}$ Thus, this study is expected to provide evidence that supports findings reported in the literature. Lopes et al. (2018) suggested that tDCS combined with a VR game for children with DS may influence brain activity by acting on local circuits. ${ }^{[56-57]}$ In another study involving tDCS combined with VR, Miziara et al. (2018) found better alpha events in the somatosensory region, especially at the assessment performed one month after the end of the intervention. Moreover, greater alpha desynchronization was found after stimulation plus training, indicating an increase in attention and concentration during the preparation of movements. To achieve better results, the authors state that it is necessary to conduct a study with a larger sample, as VR may influence the EEG signal. ${ }^{[58-60]}$ Therefore, the proposed study aims to complement the literature with further information and different variables to provide the scientific community with clinical data on this combination of interventions.

\section{Abbreviations}


AtDCS: Andal Transcranial direct current stimulation

CAPES: Coordenação de Aperfeiçoamento de Pessoal de Nível Superior

CNPq: Conselho Nacional de Desenvolvimento Científico e Tecnológico

CONSORT:

DLPFC: Dorsolateral prefrontal cortex

DS: Down syndrome

EEG: Electroencephalography

EMG: Electromyographic

MEG: Magnetoencephalography

REBEC: Brazilian Registry of Clinical Trials

SPIRIT:

TD: Tipical Development

tDCS: Transcranial direct current stimulation

VR: virtual reality

WASI: Wechsler Abbreviated Scale of Intelligence

WISC II: Wechsler Intelligence Scale for Children

\section{Declarations}

\section{Trial status}

Preparations for the development of the protocol (ethics committee, registration, registration of the protocol, submission of the protocol) will take place from JANUARY 2019 to NOVEMBER 2020.

Recruitment started in DEZEMBER 2020 with data acquisitions and protocols scheduled for 13 months and is planned to end in DEZEMBER 2022, during the execution of the protocol, it will be possible to produce partial data and, on completion, publications of the final studies.

\section{Additional file}

Additional file 1. SPIRIT 2013 Checklist: Recommended items to address in a clinical trial protocol and related documents*. 


\section{Ethics approval and consent to participate}

The present study received the REBEC protocol number (RBR-43pk59) registered on 2019/03/27 http://ensaiosclinicos.gov.br/rg/view/7875/ and approval from the Human Ethics Committee (certificate number: 3.608 .521 ) on $2019 / 09 / 30$. Any changes to the protocol will be reported to the committees and approved. Informed consents will be obtained from all participants by the clinical research coordinator and principal investigator.

\section{Consent for publication}

Not applicable.

\section{Availability of data and materials}

The methods of disseminating the original data will be reported according to the research process and the results of the study will be presented in peer-reviewed journals.

\section{Competing interests}

The authors declare that they have no competing interests.

\section{Funding}

his study is supported by a financing fund from Brazil, CNPq (National Council for Scientific and Technological Development), in the amount of R\$119,496.0, following the process number 428292 / 2018-6 on behalf of the responsible researcher Claudia Santos Oliveira.

\section{Acknowledgments}

The authors gratefully acknowledge support from the Brazilian fostering agencies Conselho Nacional de Desenvolvimento Científico e Tecnológico (CNPq [National Council of Scientific and Technological Development]), Coordenação de Aperfeiçoamento de Pessoal de Nível Superior (CAPES [Coordination for the Advancement of Higher Education Personnel]) and Fundação de Amparo á Pesquisa [Research Assistance Foundation]. The funders had no role in study design, data collection and analysis, decision to publish or preparation of the manuscript.

\section{Authors' contributions}

JBPL, IMM, DK, RBP, LVS, CBMM, DCSC, JOHM, VLSA, IOS, VO, BAC, MG, VC, and CSO all contributed to the overall design of the study, writing of the manuscript, development of the study protocol and approval of the final version of the manuscript for submission.

\section{References}


1. Bomono, LMM e Rosseti, CB. Aspectos do desenvolvimento perceptivo-motor e inteligência sensóriomotora na síndrome de down. Rev. Bras Crescimento Desenvolv Hum. 2010. 3, p. 723-734.

2. MUNDIAL DA SAÚDE. CID-10: Classificação internacional de doenças. ed. São Paulo: EdUSP, 1999.

3. Carr J. Patterns of ageing in 30-35-year-olds with Down's syndrome. Journal of Applied Research in Intellectual Disabilities. 2003. 16:29. doi.org/10.1046/j.1468-3148.2003. 00129.x.

4. Bohnen I, Strydom A. Older adults with Intellectual Disability. Current Opinion in Psychiatry; 2012. 25: 359-64.DOI: doi.org/10.1093/ageing/afy151

5. Bissoto, ML. 0 desenvolvimento cognitivo e o processo de aprendizagem do portador de Síndrome de Down: revendo concepções e perspectivas educacionais. Ciências \& Cognição. 2005; 4: p.80-88.

6. Chapman, R. S., and Hesketh, L. J. Behavioral phenotype of individuals with down syndrome. Mental Retard. Dev. Disabil. Res. Rev. 2000. 6,84-95. doi: 10.1002/1098-2779(2000)6:2\&lt;84::AIDMRDD2\&gt;3.0.CO;2-P.

7. Sveljo, O., Culic, M., Koprivesk, K., and Lucic, M. The functional neuroimaging evidence of cerebellar involvement in the simple cognitive tasks. Brain Imaging Behav. (2014). 8:4. doi: 10.1007/s11682014-9290-3.

8. Volman, M. J., Visser, J. J., and Lensvelt-Mulders, G. J. Functional status in 5 to 7-year-old children with Down syndrome in relation to motor ability and performance mental ability. Rehabil. 2007. 291, 25-31. doi: 10.1080/09638280600947617.

9. Luria AR, Tskvetkova LS. The programing of constructive activity in local brain injuries. 1964: 2; 95107. doi.org/10.1016/0028-3932(64)90015-6

10. Buckley SL, Bird G. Meeting the education needs of the children with Down syndrome. Portsmouth: Sarah Duffen Centre University of Portsmouth. doi: 10.3104 / prática.148

11. Pinter, J. D., Eliez, S., Schmitt, J. E., Capone, G. T., and Reiss, A. L. Neuroanatomy of down's syndrome: a high-resolution MRI study. Am J Psychiatry. 158, 1659-1665. doi: 10.1176/appi.ajp.158.10.1659.

12. Vygotsky LSA. Formação social da mente: o desenvolvimento dos processos psicológicos superiores. 6 ed. São Paulo. p6.

13. Grela, B. children with Down Syndrome have difficulty with argument structure? Journal of Communication Disorders, 2003. doi.org/10.1016/S0021-9924(03)00014-5

14. Cusin, DA. Avaliação do processo receptivo: investigação do desenvolvimento semântico em indivíduos com Síndrome de Down. Bras. Educação Especial. 2005. 11; p. 81-96.

15. Escamilla SG. El niño con Síndrome del Down. México: Diana, 1998.

16. Feuerstein R. Instrumental enrichment: an intervention program for cognitive modificability. Baltimore: University Park Press; 1980.

17. Clements, PR, Bates, MV \& Hafer, M. Variabilidade dentro da síndrome de Down (Trissomia do 21): diferenças sexuais empiricamente observadas no QI. Mental Retardation. 1976, 14, p. 30-31.

18. Fiedler DJ. The emerging Down Syndrome behavioral phenotype in early childhood: implications for Practice. Rev. Infants e Young Children. 2005: 18; p. 86-103. 
19. Fregni F, Bossio PS, Brunoni AR. Neuromodulação terapêutica: Princípios e avanços da estimulação cerebral não invasiva em neurologia, reabilitação, psiquiatria e neuropsicologia. São Paulo, 2012.

20. Fregni F, Gimenes R, Valle AC, Ferreira MJ, Rocha RR, Natalle L, Bravo R, Rigonatti SP, Freedman S, Nitsche M, Pascual-Leone A, Boggio PS. A randomized, sham-controlled, proof of principle study of transcranial direct current stimulation for the treatment of pain in fibromyalgia. Arthritis and Rheumatism. 2006: 54;3988-98. doi:10.1002 / art.22195.

21. Mendonça ME, Fregni F. Neuromodulação com estimulação cerebral não invasiva: aplicação no acidente vascular encefálico, doença de Parkinson e dor crônica. In.:ASSIS, R.D. Condutas práticas em fisioterapia neurológica. Manole. São Paulo, 2012. p. 307-39.

22. Miranda PC, Lomarev $\mathrm{M}$, Hallett $\mathrm{M}$. Modeling the current distribution during transcranial direct current stimulation. Clin Neurophysiol. 2006: 117(7);1623-9. doi: 10.1016 / j.clinph.2006.04.009.

23. Creutzfeldt OD, Fromm GH, Kapp H. Influence of transcortical d-c currents on cortical neuronal activity. Exp Neurol. 1962: 5;436-52.10.1016 / 0014-4886 (62) 90056-0

24. Goldring S, OهLeary JL. Summation of certain enduring sequelae of cortical activation in the rabbit. Electroencephalogr Clin Neurophysiol. 1951: 3(3); p. 329-40. doi.org/10.1016/0013-4694(51)900818.

25. Nitsche MA, Paulus W. Sustained excitability elevations induced by transcranial DC motor cortex stimulation in humans. Neurology. 2001: 27(10);1899-901. doi: 10.1212 / wnl.57.10.1899.

26. Wagner T, Fregni F, Fecteau S, Grodzinsky A, Zahn M, Pascual-Leone A. Transcranial direct current stimulation: A computer-based human model study. Neuroimage 2007. doi: 35:1113-24. 10.1016 / j.neuroimage.2007.01.027

27. Mello B, Ramalho $T$. Use of virtual reality in the physical therapeutic treatment of individuals with down syndrome. Revista Neurociências 2015; 23: 143-9. doi: 10.4181 / RNC.2015.23.01.985.7p.

28. McCarthy, M, Silberstein, CE, Atkins, EA, Harryman SE, Sponseller, PD, Miller NAH. Comparing reliability and validity of pediatric instruments for measuring health and well-being of children with spastic cerebral palsy. Developmental Medicine\& Child Neurology.2002; 44: 468-476. doi:

$10.1017 /$ s0012162201002377

29. Nitsche MA, Paulus W. Sustained excitability elevations induced by transcranial DC motor cortex stimulation in humans. Neurology. 2001: 27(10);1899-901. doi.org/10.1212/WNL.57.10.1899

30. Wagner T, Fregni F, Fecteau S, Grodzinsky A, Zahn M, Pascual-Leone A. Transcranial direct current stimulation: A computer-based human model study. Neuroimage 2007; 35:1113-24. doi: 10.1016 / j.neuroimage.2007.01.027

31. Toshida K., limoto Y., Ando S. A biomechanical study on movement of forehand top spin stroke in table tennis. International Journal of Table Tennis Sciences 1996 3, 28.

32. Monte-Silva K, Kuo MF, Thirugnanasambandam N,Liebetanz D, Paulus W, Nitsche MA. Dosedependent inverted $U$-shaped effect of dopamine (D2-like) receptor activation on focal and nonfocal plasticity in humans. The Journal of Neuroscience. 2009;29 (19):6124-31. doi: 10.1523 / JNEUROSCI.0728-09.2009 
33. Liebetanz D, Nitsche MA, Teragau F, Paulus W. Pharmacological approach a to the mechanisms of transcranial DC- stimulation- induced after-effects of human motor cortex excitability. 2002;125(10):2238-47. doi:10.1093 / brain / awf238.

34. Nitsche MA, Liebetanz D, Schlitterlau a, Henschke U, Friche K, Frommann K, et al. GABAergic modulation of DC stimulation-induced motor cortex excitability shifts in humans. Eur J Neurosco. 2004; 19:2720-6. doi: 10.1111/j.0953-816X.2004.03398.x.

35. Delorme A. Makeig S. EEGLAB: an open source toolbox for analysis of single-trial EEG dynamics including independent component analysis. Journal of Neuroscience Methods. 2004; 134: 9-21. doi: 10.1016 / j.jneumeth.2003.10.009.

36. Tommaso Md, Ricci K, Delussi $\mathrm{M}$ et al. Testing a novel method for improving wayfinding by means of a P3b Virtual Reality Visual Paradigm in normal aging. 2016 SpringerPlus 5:1297. doi: 10.1186 / s40064-016-2978-7

37. Hermens H, Freriks B, Merletti R et al. Raccomandazioni Europee per l'Elettromiografia di Superficie. Turin; CLUT Editrice 2000

38. Cimolin V, Beretta E, Piccinini L, et al. Constraint-induced movement therapy for children with hemiplegia after traumatic brain injury: a quantitative study. J Head Trauma Rehabil 2012; 27:17787. doi: 10.1097 / HTR.0b013e3182172276

39. Menegoni F, Milano E, Trotti C, et al. Quantitative evaluation of functional limitation of upper limb movements in subjects affected by ataxia. Eur J Neurol 2009; 16:232-9. doi:10.1111 / j.14681331.2008.02396.x

40. Petuskey K, Bagley A, Abdala E, et al. Upper extremity kinematics during functional activities: threedimensional studies in a normal pediatric population. Gait Posture 2007; 25:573-9. doi: 10.1016 / j.gaitpost.2006.06.006

41. Rab G, Petuskey K, Bagley A. A method for determination of upper extremity kinematics. Gait Posture 2002; 15:113-9. doi: 10.1016 / s0966-6362 (01) 00155-2

42. Moura RFC, Grecco LAC, Santos C, Lazarri RD, Duarte NC, Lopes JBP. Transcranial direct current stimulation combined with upper limb functional training in children with spastic, hemiparetic cerebral palsy: study protocol for a randomized controlled trial. 2016 Clinical Trials. doi: 10.1186 / s13063-016-1534-7

43. Pavão SL, Arnoni JLB, Oliveira AKC. Impacto de Intervenção em realidade virtual sobre o desempenho motor de uma criança com paralisia cerebral: estudo de caso Revista paulista de pediatria. 2014;32: 389-394. doi.org/10.1590/S0103-05822014000400016

44. Brunamonti E, Pani P, Papazachariadis O, Onorati, P, Albertini G, Ferraina S. Cognitive control of movement in down syndrome. Res Dev Disabil.2011, 32(5):1792-1797. doi.org/10.1016/j.ridd.2011.03.008.

45. Grecco LAC, Duarte NAC, Mendonça M, Zanon N, Fregni F, Oliveira CS. Transcranial direct current stimulation combined with treadmill gait training in delayed neuro psychomotor development. Journal of Physical Therapy Science 2014; 26:945-950 
46. Grecco LA, de Almeida Carvalho Duarte N, Mendonça ME, Cimolin V, Galli M, Fregni F, Santos Oliveira C. Transcranial direct current stimulation during treadmill training in children with cerebral palsy: a randomized controlled double-blind clinical trial. Res Dev Disabil. 2014. Nov;35(11):2840-8. doi: 10.1016/j.ridd.2014.07.030. Epub 2014 Aug 6.

47. Grecco LA, Duarte NA, Zanon N, et al. Effect of a single session of transcranial direct current stimulation on balance and spatiotemporal gait variables in children with cerebral palsy: A randomized sham-controlled study. Braz J Phys Ther. 2014; Sep-Oct;18(5): 419-

27.doi.org/10.1590/bjpt-rbf.2014.0053

48. Duarte NA, Grecco LA, Galli M, Fregni F, Oliveira CS. Effect of transcranial direct-current stimulation combined with treadmill training on balance and functional performance in children with cerebral palsy: a double-blind randomized controlled trial. PLoSOne. 2014 Aug29;9(8): e105777.doi:

10.1371/journal.pone.0105777. eCollection2014

49. Gillick T, Feyma T., Menk J., Usset M., Vaith A., Wood J., WorthingR., Krach E. Safety and Feasibility of Transcranial Direct Current Stimulation in Pediatric Hemiparesis: Randomized Controlled Preliminary Study. Physical Therapy 2014;Nov 20. Doi:10.2522 / ptj.20130565

50. Chandramouli Krishnan, Luciana Santos, Mark D. Peterson, Margaret Ehinger, Safety of Noninvasive Brain Stimulation in Children and Adolescents. Brain Stimulation.2015. 8 : 76-87. doi: 1016 / j.brs.2014.10.012

51. Choi, J. T., Jensen, P., \& Nielsen, J. B. (2016). Locomotor sequence learning in visually guided walking. Journal of Neurophysiology, 115(4), 2014-2020. doi: 10.1152 / jn.00938.2015

52. Hupfeld, K. E., Ketcham, C. J., \& Schneider, H. D. (2017). Transcranial direct current stimulation (tDCS) to the supplementary motor area (SMA) influences performance on motor tasks. Experimental Brain Research, 235(3), 851-859 doi: 10.1007 / s00221-016-4848-5

53. Monteiro CBM, Silva TD, Abreu LC, Fregni F, Viera L, Ferreira FHIB, Leone C. Short-term motor learning through non-immersive virtual reality task in individuals with down syndrome. BMC Neurol. 2017 14;71. doi: 10.1186/s12883-017-0852-z.

54. Damian S, Pradeep R, Amir T, Jitendra J, Suchada V, Assessing Video Games to Improve Driving Skills: A Literature Review and Observational Study. JMIR Serious Games. 2014. ;2(2):5. doi:10.2196/games.3274

55. Biddiss E, Beng Jl. Active Video Games to Promote Physical Activity in Children and Youth A Systematic Review. Arch pediatr adolesc med. 2010. doi: 10.1001 / archpediatrics.2010.104.

56. Lopes JBP, Grecco LAC, Moura RCF, Lazarri RD, Duarte NAC, Miziara IM et al. Protocol study for a randomised, controlled, double-blind, clinical trial involving virtual reality and anodal transcranial direct current stimulation for the improvement of upper limb motor function in children with Down syndrome. BMJ Open 2017. DOI: 10.1136 / bmjopen-2017-016260

57. Lopes JBP, Miziara IM, Galli, M, Cimolin V, Oliveira CS. Effect of Transcranial Direct Current Stimulation Combined With Xbox-kinect Game Experience on Upper Limb Movement in Down 
Syndrome: A Case Report. Frontiers in Bioengineering and Biotechnology, v. 1, p. 1, 2020. doi: 3389 / fbioe.2020.00514 PMCID: PMC7273846

58. Lopes, JBP, Miziara IM, Kahani, D, Conway B, OLIVEIRA CS. Electroencephalographic analysis of brain activity after interventions with transcranial direct current stimulation over the motor cortex: $\mathrm{A}$ systematic review. Adaptive Behavior, v. 1, p. 1-17, 2020.Doi: 10.1177/1059712320930753

59. Lopes JBP, Miziara IM, Moura RCF, Lazarri RD, Fonseca P, Dumont AJL. et al. Effect of transcranial direct current stimulation on motor control during reaching movement in a child with down syndrome - case report. GAIT \& POSTURE. doi.org/10.1016/j.gaitpost.2018.07.037.

60. Miziara IM, Lopes JBP, Kahani D, Lazzari R, De Moura RCF, Conway, BA et al. Analysis of eeg signal in a child with hemiparetic cerebral palsy during a motor activity: Case study. GAIT \& POSTURE 2018 1; 1-1. doi.org/10.1016/j.gaitpost.2018.07.036.

\section{Figures}




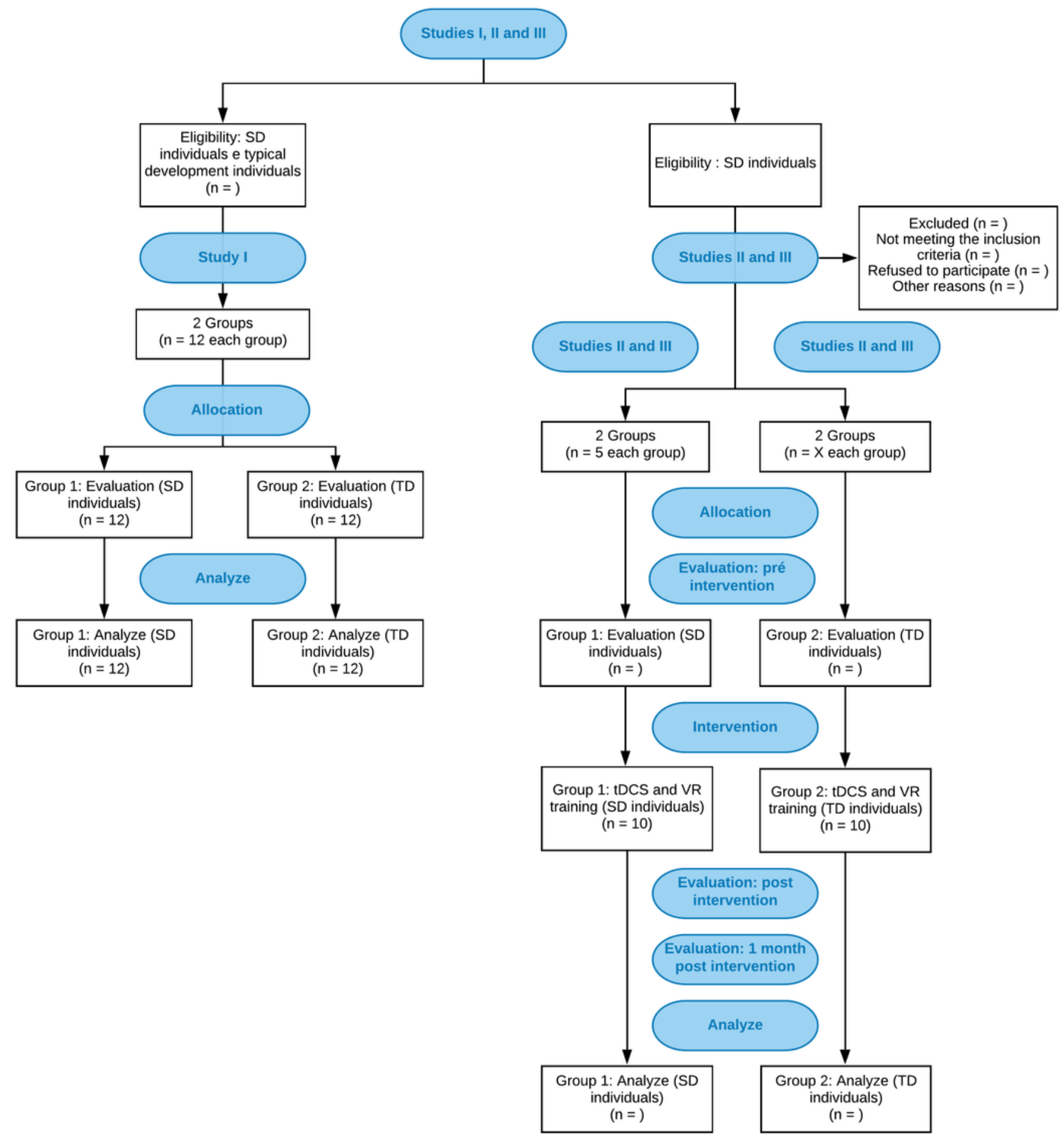

\section{Figure 1}

Flowchart of phases according to the CONSORT statement. DS - Down syndrome; TD - typical development; tDCS - transcranial direct current stimulation; VR - virtual reality. 

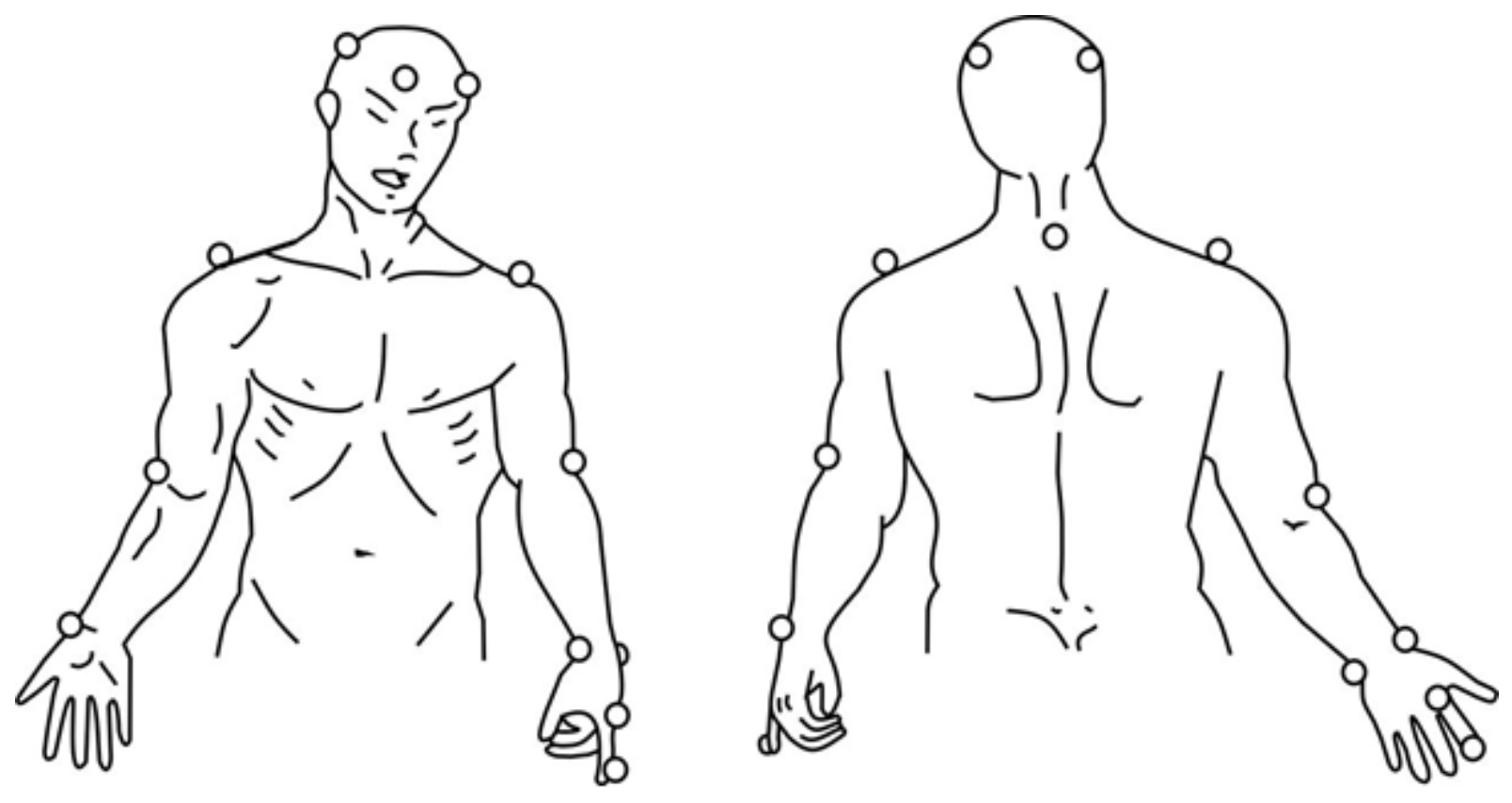

Figure 2

Placement of markers for three-dimensional analysis using SMARTup: the experimental setup. 


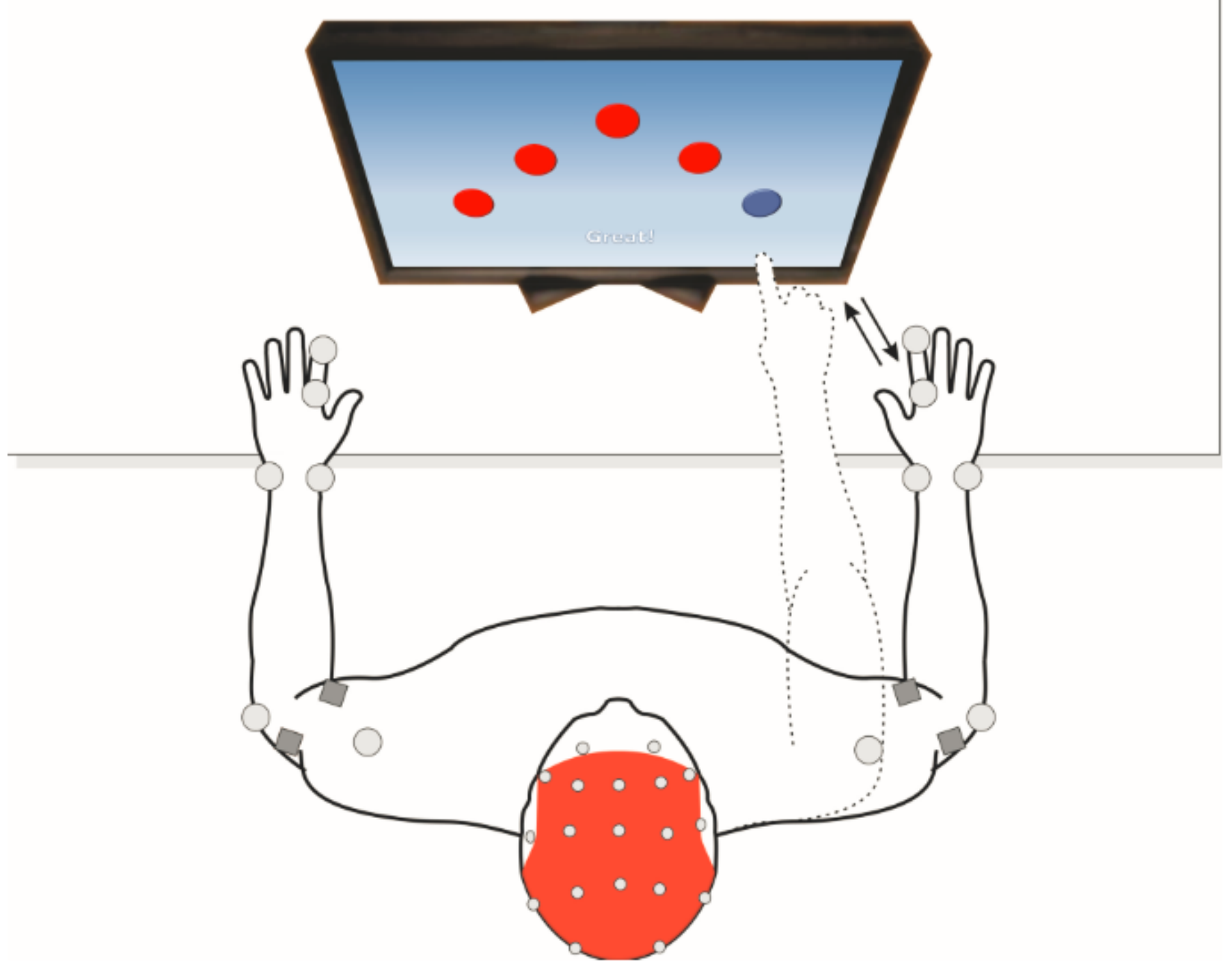

Figure 3

Equipment and execution of reaching/cognitive task. 


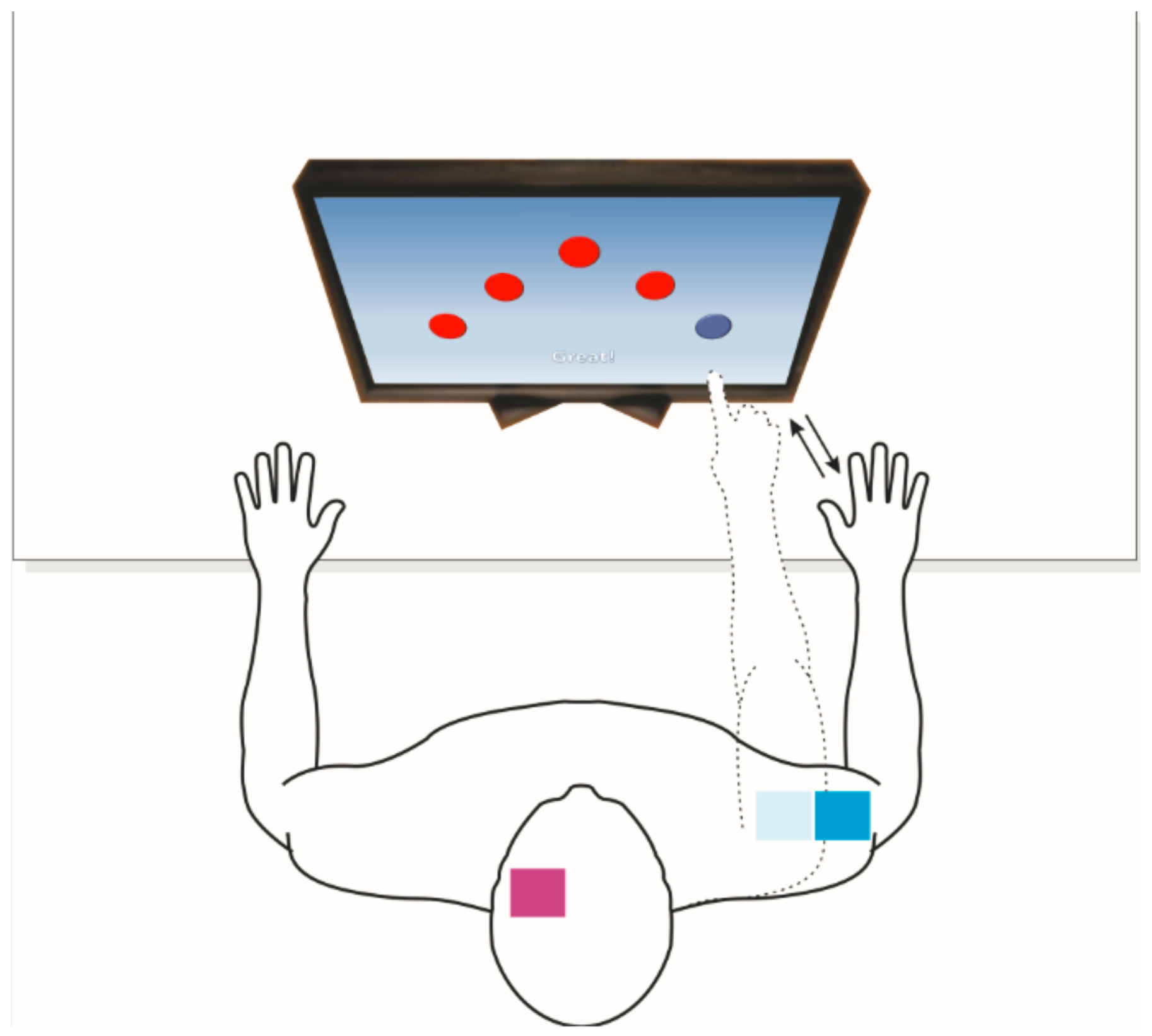

Figure 4

Anode (pink) and cathode (blue) placement and virtual reality training. 